\title{
PROPAGACION in vitro DE SEGMENTOS NODALES DE CEDRO (Cedrela odorata L.) OBTENIDOS A PARTIR DE SEMILLAS BOTANICAS
}

\author{
Ximena HUAMÁN ${ }^{1}$, María Emilia RUIZ-SÁNCHEZ1 ${ }^{1}$ Juan Carlos GUERRERO-ABAD², Roger PICHIS- \\ GARCÍA $^{2}$, Lucas GARCÍA², Reynaldo SOLIS ${ }^{2}$ \\ 1 Universidad Nacional de San Martín - Tarapoto (UNSM-T). Laboratorio de Cultivo de Tejidos Vegetales, Facultad de \\ Ciencias Agrarias. San Martín, Perú. E-mail: ximena18h@hotmail.com \\ 2 Instituto de Investigaciones de la Amazonía Peruana (IIAP). Programa de Investigación en Manejo Integral del Bosque y \\ Servicios Ambientales (PROBOSQUES). Jr. Belén Torres de Tello N¹35, Morales, San Martín, Perú.
}

\begin{abstract}
RESUMEN
El objetivo de este trabajo fue optimizar la germinación de semillas botánicas en condiciones in vitro y establecer un medio de cultivo que nos permita la multiplicación y enraizamiento de plántulas a partir de segmentos nodales. La metodología comprendió la desinfección de las semillas botánicas in vitro, para lo cual fueron sumergidas en alcohol 70\% durante 60 segundos y enjuagadas 3 veces con agua destilada estéril, luego fueron sumergidas en $\mathrm{NaOCl} 1.5 \%$ durante 10 y 15 minutos por cada tratamiento y enjuagadas 3 veces con agua destilada estéril. Los segmentos nodales obtenidos de las plantas que germinaron a partir de semillas botánicas en condiciones in vitro fueron sub cultivadas en medio de cultivo MS suplementado con diferentes concentraciones de reguladores de crecimiento: $\operatorname{BAP}\left(0.5,1\right.$ y $\left.2 \mathrm{mg} . \mathrm{L}^{-1}\right)$ y ANA $\left(0,0.1\right.$ y $\left.0.25 \mathrm{mg} . \mathrm{L}^{-1}\right)$. Los resultados muestran que las semillas botánicas tratadas con alcohol 70\% (60 segundos) y NaOCl 1.5\% (15 minutos) presentaron menor porcentaje de contaminación (42.22\%) y un mayor porcentaje de germinación (76\%). En el caso de segmentos nodales, se obtuvieron mejores resultados en aquellos cultivados en MS suplementado con $0.5 \mathrm{mg} . \mathrm{L}^{-1}$ de BAP y $0.1 \mathrm{mg} . \mathrm{L}^{-1}$ de ANA (plantas de $4.82 \mathrm{~cm}$ con un coeficiente de multiplicación de 2.87). Con este mismo medio se obtuvo un $100 \%$ de enraizamiento con un promedio 8.13 raíces por planta a las 5 semanas de individualizada, permitiendo obtener semilla vegetativa de calidad.
\end{abstract}

PALABRAS CLAVE: Cedrela odorata, in vitro, reguladores de crecimiento, segmentos nodales, enraizamiento.

\section{In vitro PROPAGATION OF NODAL SEGMENTS OF CEDAR (Cedrela odorata L.) OBTAINED FROM BOTANICAL SEEDS}

\begin{abstract}
The objective of this study was to optimize the in vitro germination of botanical seeds and establish the conditions and cultivation medium for its multiplication and rooting from nodal segments. For the disinfection of botanical seeds in the in vitro establishment, these were immersed in $70 \%$ alcohol for 60 seconds and rinsed 3 times with sterile distilled water and then they were immersed in $1.5 \% \mathrm{NaOCl}$ for 10 and 15 minutes per treatment and rinsed 3 times with sterile distilled water. The nodal segments obtained from the plants that germinated from botanical seeds in in vitro conditions were sub cultivated on MS medium supplemented with different concentrations of growth regulators: BAP $(0.5,1$ and $2 \mathrm{mg} . \mathrm{L}-1)$ and NAA $(0,0.1$ and $0.25 \mathrm{mg} . \mathrm{L}-1)$. Botanical seeds treated with alcohol $70 \%$ (60 seconds) and $1.5 \% \mathrm{NaOCl}$ (15 minutes) had lower contamination rate (42.22\%) and a higher percentage of germination (76\%). The MS medium supplemented with $0.5 \mathrm{mg} . \mathrm{L}-1$ of BAP and $0.1 \mathrm{mg} \mathrm{L}-1$ of ANA allowed the development of seedlings of 4.82 $\mathrm{cm}$ with a multiplication factor of 2.87 . Furthermore using the same culture medium was obtained $100 \%$ rooting with 8.13 roots per plant at 5 weeks of individualized, obtaining high quality vegetative seeds.
\end{abstract}

KEYWORDS: Cedrela odorata, in vitro, growth regulators, nodal segments, rooting. 


\section{INTRODUCCIÓN}

La especie de mayor importancia comercial en la Amazonía es el cedro Cedrela odorata L., esta especie es muy apreciada en el mercado internacional por su durabilidad, peso liviano, color atractivo y resistencia al ataque de termitas (Muñoz, 2003). En los últimos años el cedro está sufriendo una fuerte disminución del tamaño de sus poblaciones naturales ocasionado por la sobreexplotación, la destrucción de su hábitat y la falta de tecnologías para su reproducción. La Unión Internacional para la Conservación de la Naturaleza (IUCN, 2004) lista a Cedrela odorata L. como una especie que enfrenta un alto riesgo de extinción en estado silvestre a mediano plazo. También está incluida en el Apéndice III de la lista de especies prioritarias de la Convención para el Comercio Internacional de Especies amenazadas de fauna y flora Silvestre -CITES.

Ante este panorama diversas instituciones del estado vienen promoviendo su reforestación y establecimiento de plantaciones a partir de propagación natural o propagación vegetativa. No obstante la propagación natural presenta una área de dispersión corta y ocurre estacionalmente por medio de semillas (Daquinta et al., 2004), estos eventos son importantes para mantener una amplia base genética. En la propagación vegetativa de esta especie se ha realizado avances significativos generándose metodologías de enraizamiento de estacas juveniles, consiguiéndose hasta $93.3 \%$ de estacas juveniles enraizadas en propagación en cámaras de sub irrigación (Murrieta, 2010). Una de las desventajas de la propagación por enraizamiento de estacas es que las plantas obtenidas no pueden seguir siendo multiplicadas en procesos sucesivos, además que no todas las especies producen rebrotes. Otra de las problemáticas del cedro es que en sus plantaciones son afectadas por las larvas de la mariposa Hypsiphylla grandella, tanto en etapa de vivero, como durante su adaptación y crecimiento en campo (Jiménez-Terry et al., 2007). La larva se alimenta de la yema apical destruyéndola, su acción produce la deformación y bifurcación del tronco retrasando considerablemente el crecimiento de la planta afectada y ocasionalmente puede causar la muerte (Patiño, 1997). Afortunadamente la especie presenta una gran variabilidad genética en la Amazonía peruana (De la Torre et al., 2008) y es posible encontrar individuos con tolerancia a este insecto. Aunque la literatura reporta escasos reportes de la existencia de diferentes formas de resistencia en Meliaceae al barrenador de la yema Hypsiphylla grandella, con particular énfasis en los trabajos desarrollados en Costa Rica con Cedrela odorata y
Swietenia macrophylla muestra significativa existencia de resistencia genética al barrenador en estas especies arbóreas (Patiño, 1997).

En los últimos años las técnicas biotecnológicas han contribuido a la generación de una metodología de desarrollo in vitro, así como avances en estudios de propagación vía organogénesis (Pérez et al., 2002; Pérez et al. 2006; García-Gonzáles et al., 2011) y embriogénesis somática (Muñoz, 2003; Daquinta et al., 2004; Cameron, 2010) de Cedrela odorata. También se han realizado estudios en la propagación in vitro de Cedrela fissilis (Da Costa-Nunes et al., 2001; Vila et al., 2009). Los principales problemas que se han presentado en las investigaciones en propagación in vitro de cedro son: la contaminación de las yemas apicales y segmentos nodales, la hiperhidricidad, la poca disponibilidad de explantes, la sensibilidad a la desinfección y la lenta brotación (Jiménez-Terry et al., 2007).

La obtención de plantas de cedro a partir de semillas de genotipos élite y el uso del cultivo in vitro para su propagación constituye una alternativa importante para la producción de grandes volúmenes de plantas a corto plazo y así poder suministrar semilla vegetativa de alta calidad genética en el establecimiento de plantaciones comerciales. Por lo anterior, el objetivo de este trabajo fue germinar in vitro semillas de cedro y establecer un medio de cultivo que nos permita la multiplicación y enraizamiento in vitro de plantas a partir de segmentos nodales.

\section{MATERIALES Y MÉTODOS}

El presente trabajo se realizó en el laboratorio de cultivo de tejidos vegetales de la Universidad Nacional de San Martín - Tarapoto. Las semillas botánicas de cedro fueron colectadas del campo de multiplicación de Bello Horizonte, distrito de Banda del Shilcayo, región San Martin (18M 9281954, 0357544).

Para la desinfección de las semillas botánicas in vitro, estas fueron sumergidas en alcohol $70 \%$ durante 60 segundos y enjuagadas tres veces con agua destilada estéril, luego fueron sumergidas en $\mathrm{NaOCl} 1.5 \%$ durante (10 y 15 minutos) y enjuagadas tres veces con agua destilada estéril. Las semillas fueron establecidas en un medio nutritivo Murashige y Skoog (Murashige \& Skoog, 1962) a mitad de concentración (MS/2). Las variables evaluadas fueron: porcentaje de contaminación y porcentaje de germinación.

Los segmentos nodales obtenidos de las plantas germinadas fueron sub cultivadas en medio de cultivo MS/2 suplementado con carbón activado (2 g. $\left.\mathrm{L}^{-1}\right)$, agar $\left(10\right.$ g. $\left.\mathrm{L}^{-1}\right)$ y tres concentraciones de 
reguladores de crecimiento: $\mathrm{BAP}\left(0.5,1\right.$ y $\left.2 \mathrm{mg} . \mathrm{L}^{-1}\right)$ y dos concentraciones de ANA ( $0,0.1$ y $\left.0.25 \mathrm{mg} . \mathrm{L}^{-1}\right)$. Los tratamientos empleados se indican en la Tabla 1. $\mathrm{El} \mathrm{pH}$ del medio de cultivo fue ajustado a 5.65 y las variables evaluadas fueron: altura de plantas, número de nudos, porcentaje de enraizamiento, número y longitud de raíces. La evaluación se realizó 5 semanas después de la siembra de los segmentos nodales.

Los datos fueron analizados mediante el análisis de varianza y sometidos a la prueba de Duncan $(\mathrm{p}<$ $0,01)$ para determinar la naturaleza de las diferencias entre los tratamientos. Se empleó el programa estadístico Infostat/Pversión 1.1.

\section{RESULTADOS Y DISCUSIÓN}

\section{ESTABLECIMIENTO in vitro DESEMILLAS BOTÁNICAS}

La contaminación es uno de los principales problemas a resolver en el cultivo in vitro. Los contaminantes pueden originarse de dos fuentes distintas: aquellos que vienen en la superficie o en los tejidos de la planta donadora y aquellos que surgen como resultados de fallas en los procedimientos de laboratorio.

La presencia de agentes contaminantes afectan el normal desarrollo de los explantes establecidos a condiciones in vitro haciendo indispensable el uso de metodologías que permitan la desinfección. En el presente estudio, cuando se incrementó el tiempo de exposición de $\mathrm{NaOCl} 1.5 \%$ de 10 a 15 minutos durante el establecimiento in vitro el porcentaje de contaminación disminuye y el porcentaje de germinación aumenta (Tabla 2), observándose que la contaminación inicial por hongos y bacterias influyó negativamente en la germinación in vitro de semillas de cedro tal como lo observó Pérez et al. (2002). Esto se puede atribuir a la asociación de las semillas de especies forestales tropicales con muchos microorganismos que de manera natural ayudan en los procesos de germinación pero que representan un factor limitante para los trabajos in vitro (Pérez et al., 2002).

En los trabajos realizados con ápices y segmentos nodales de cedro, la exposición de estos explantes a $\mathrm{NaOCl} 1.5 \%$ durante 10 minutos permitió alcanzar el valor máximo de supervivencia y un bajo porcentaje de contaminación. Al incrementar el tiempo de exposición en 15 minutos el valor de ambas variables disminuyen ligeramente (JiménezTerry et al., 2007). A su vez García-Gonzáles et al. (2011) establecieron que lavados de segmentos nodales de cedro con propiconazol CE 25 al $5 \%$ durante 3 minutos posibilita un $100 \%$ de desinfección de estos explantes.

\section{EFECTO DEL BAPYEL ANA EN LA PROPAGACION in vitro}

En la obtención de plantas in vitro de cedro obtener un buen tamaño y follaje confieren a la planta mayores posibilidades de sobrevivir durante la aclimatación en los invernaderos, debido a que estos dos parámetros están relacionados con una mayor capacidad fotosintética. En tanto que plantas con mayor número de nudos permite tener un mayor coeficiente de multiplicación.

Nuestros resultados muestran que si bien las diferentes concentraciones de los reguladores de crecimiento BAP y ANA, influenciaron igualmente en el crecimiento in vitro del cedro, lográndose plantas completas y buen desarrollo radicular. Sin embargo el tratamiento T2 $\left(0.5 \mathrm{mg} . \mathrm{L}^{-1}\right.$ de BAP y 0.1 $\mathrm{mg} . \mathrm{L}^{-1}$ de ANA) indujo la formación de plantas de mayor altura, con buen número de nudos y $100 \%$ de formación de raíces bien desarrolladas, con diferencias significativas frente al resto de tratamientos (Tabla 3).

Igualmente todas las concentraciones de BAP empleadas resultaron eficaces para la brotación de segmentos nodales. Si bien el tratamiento T4 (1 $\mathrm{mg} . \mathrm{L}^{-1}$ de BAP), presento plantas con mayor número de nudos y fue numéricamente superior que el resto de los tratamientos, pero estas diferencias no fueron significativas con los resultados obtenidos en el resto de tratamientos. No obstante el tratamiento T4 presento numéricamente mayor número de nudos, pero también presento menor altura de las plantas que T2, por lo tanto los segmentos nodales fueron más cortos. Entonces el tratamiento T2 es más recomendable cuando comparado con $\mathrm{T} 4$, porque reducen los costos de propagación y las posibilidades de desarrollar variantes somaclonales. En la figura 1 muestra las plántulas obtenidas en T2.

Estudios realizados en la especie reportan que concentraciones de 0.5 y $1.5 \mathrm{mg} . \mathrm{L}^{-1}$ de BAP favorece la brote de los segmentos nodales (Pérez et al., 2002), sin embargo el tamaño de los brotes obtenidos $(1 \mathrm{~cm})$ no garantiza el éxito para pasar a la fase posterior del cultivo in vitro por su difícil manejo y mayor susceptibilidad a la manipulación. La adición de $2 \mathrm{mg} . \mathrm{L}^{-1}$ de BAP y $3 \mathrm{mg} . \mathrm{L}^{-1}$ de ANA al medio basal MS propició la brotación del $100 \%$ de segmentos nodales y la formación de plantas con una altura promedio de $3.93 \mathrm{~cm}$ a las 8 semanas (GarcíaGonzáles et al., 2011). Mientras que Muñoz (2003) indica que la tasa de multiplicación de cedro se puede cuantificar en base a 4 yemas por plántula en intervalos de 5 meses. Estos datos contrastan con los resultados obtenidos en este estudio ya que en T2 se obtuvieron plantas de $4.82 \mathrm{~cm}$ y 2.87 nudos en 5 semanas de cultivo. La literatura muestra también que la adición de $20 \%$ de agua de coco en el medio 
TY17 incrementa el número de brotes adventicios a partir de segmentos de hipocotilo de cedro con un número promedio de 4.68 brotes por explante (González-Rodríguez \& Peña-Ramirez, 2007; PeñaRamírez et al., 2010). Asimismo trabajos realizados con Cedrela fissilis muestran que la adición de BAP al medio de cultivo es indispensable para la brotación y multiplicación de brotes axilares ( $\mathrm{Da}$ Costa-Nunes et al., 2001).

Durante el cultivo in vitro las plantas crecen bajo un ambiente con alta humedad relativa, baja intensidad luminosa, temperatura constante, escaso intercambio gaseoso y medios ricos en compuestos orgánicos, especialmente sacarosa. Estas condiciones provocan cambios en la morfología y la fisiología de las plantas, que las hacen diferir de las que crecen en invernaderos o en el campo (Agramonte et al., 1998). Para lograr que las plantas micropropagadas sobrevivan al proceso de aclimatación y transplante a campo definitivo es necesario que estas tengan un buen número y tamaño de raíces. En nuestro estudio, al evaluar el desarrollo radicular de las plantas se observó que todos los tratamientos presentaron altos porcentajes de enraizamiento. El tratamiento T2 presenta $100 \%$ de enraizamiento y mayor número de raíces con diferencias significativas frente al resto de tratamientos, mientras que en la variable longitud de raíces los tratamientos T2, T5 y T8 no presentan diferencias significativas entre ellas pero son estadísticamente superiores frente al resto de tratamientos (Tabla 3). El estudio realizado por García-Gonzáles et al. (2011) muestran que la misma concentración que favoreció el desarrollo de los segmentos nodales también propició la formación de raíces pero ellos obtuvieron 3.9 raíces por planta a las 6 semanas de individualizada mientras que en este estudio se obtuvo 8.13 raíces por planta 5 semanas después de iniciado el cultivo. El empleo del mismo medio de cultivo para multiplicar y enraizar los explantes permitirá abaratar los costos de propagación. Los resultados obtenidos nos permitirán continuar con los estudios en propagación clonal y poder contribuir al suministro de semilla vegetativa de alta calidad genética y fitosanitaria de esta especie de vital importancia en la Amazonía Peruana.

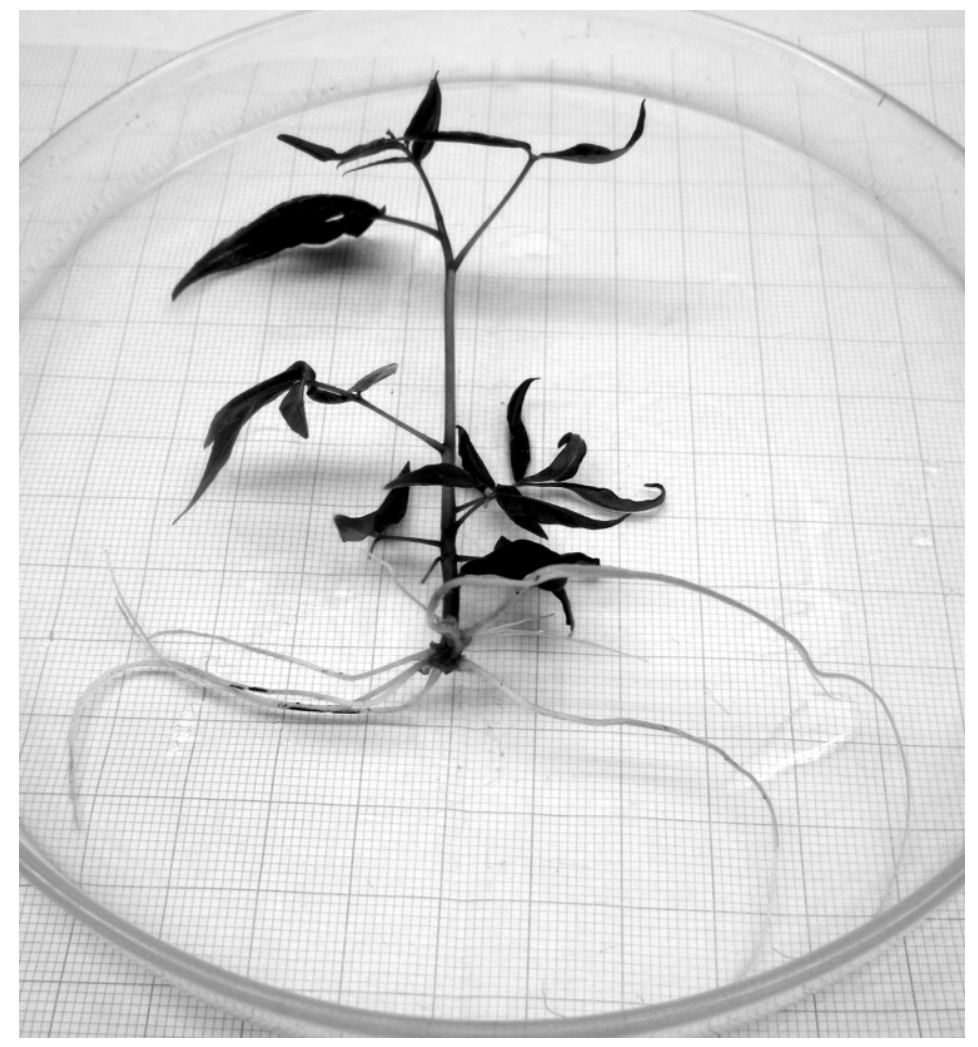

Figura 1. Planta in vitro de Cedrela odorata cultivada en el tratamiento T2, obtenida 5 semanas después de la siembra en el medio de cultivo. 
Tabla 1. Tratamientos empleados en la propagación in vitro de cedro (Cedrela odorata L.).

\begin{tabular}{ccc}
\hline Tratamiento & $\begin{array}{c}\text { Concentración de BAP } \\
\text { (mg.L-1) }\end{array}$ & $\begin{array}{c}\text { Concentración de ANA } \\
\text { (mg.L-1) }\end{array}$ \\
\hline T1 & 0.5 & 0.5 \\
T2 & 0.5 & 0.5 \\
T3 & 0.5 & 0.5 \\
T4 & 1 & 1 \\
T5 & 1 & 1 \\
T6 & 1 & 1 \\
T7 & 2 & 2 \\
T8 & 2 & 2 \\
T9 & 2 & 2 \\
\hline
\end{tabular}

Tabla 2. Efecto de los tratamientos de desinfección en el establecimiento in vitro de semillas de cedro (Cedrela odorata L.)

\begin{tabular}{ccc}
\hline Tratamientos & $\begin{array}{c}\text { Porcentaje de } \\
\text { contaminación (\%) }\end{array}$ & $\begin{array}{c}\text { Porcentaje de } \\
\text { germinación (\%) }\end{array}$ \\
\hline T1: Alcohol 70 \% (60 segundos) y NaOCl 1,5\% (10 minutos). & 66,66 & 66,66 \\
T2: Alcohol 70\% (60 segundos) y NaOCl 1,5\% (15 minutos). & 42,2 & 76 \\
\hline
\end{tabular}

Tabla 3. Efecto del BAP y el ANA en la propagación in vitro de cedro (Cedrela odorata L.).

\begin{tabular}{cccccc}
\hline Tratamiento & $\begin{array}{c}\text { Altura de } \\
\text { planta }(\mathbf{c m})\end{array}$ & $\begin{array}{c}\text { Número } \\
\text { de nudos }\end{array}$ & $\begin{array}{c}\text { Enraizamiento } \\
(\%)\end{array}$ & $\begin{array}{c}\text { Número } \\
\text { de raíces }\end{array}$ & $\begin{array}{c}\text { Longitud de } \\
\text { raíces }(\mathbf{c m})\end{array}$ \\
\hline $\mathrm{T} 1$ & $4.49 \mathrm{ab}$ & $2.67 \mathrm{bc}$ & 93.3 & $5.87 \mathrm{~cd}$ & $9.54 \mathrm{ab}$ \\
$\mathrm{T} 2$ & $4.82 \mathrm{a}$ & $2.87 \mathrm{abc}$ & 100 & $8.13 \mathrm{a}$ & $10.43 \mathrm{a}$ \\
$\mathrm{T} 3$ & $3.70 \mathrm{~cd}$ & $2.6 \mathrm{bc}$ & 100 & $.27 \mathrm{de}$ & $9.39 \mathrm{ab}$ \\
$\mathrm{T} 4$ & $4 \mathrm{bc}$ & $3.25 \mathrm{a}$ & 86.6 & $6.63 \mathrm{bc}$ & $6.78 \mathrm{~d}$ \\
$\mathrm{~T} 5$ & $4.29 \mathrm{abc}$ & $3 \mathrm{ab}$ & 93.3 & $4.47 \mathrm{ef}$ & $10.55 \mathrm{a}$ \\
$\mathrm{T} 6$ & $3.97 \mathrm{bc}$ & $2.47 \mathrm{c}$ & 100 & $6.20 \mathrm{bc}$ & $7.29 \mathrm{~cd}$ \\
$\mathrm{~T} 7$ & $3.89 \mathrm{bcd}$ & $2.58 \mathrm{bc}$ & 86.6 & $6.75 \mathrm{~b}$ & $8.70 \mathrm{~b}$ \\
$\mathrm{~T} 8$ & $3.37 \mathrm{~d}$ & $2.67 \mathrm{bc}$ & 100 & $4 \mathrm{f}$ & $9.35 \mathrm{ab}$ \\
$\mathrm{T} 9$ & $4.37 \mathrm{ab}$ & $2.87 \mathrm{abc}$ & 100 & $4.93 \mathrm{e}$ & $8.39 \mathrm{bc}$ \\
\hline
\end{tabular}

${ }^{*}$ Medias con diferentes letras en una misma columna difieren estadísticamente según la Prueba de Duncan $(p<0,01)$. 


\section{BIBLIOGRAFÍA CITADA}

Agramonte, D.; Jiménez, F.; Dita, M.A. 1998. Aclimatización. In: Pérez Ponce, J.N. (Ed). Propagación y Mejora de Plantas por Biotecnología. p. 193-206.

IUCN. 2004. Americas Regional Workshop on Conservation and Sustainable Management of Trees (Cedrela odorata). In IUCN Red list of threatened species. http://www.redlist.org.

Cameron, S.I. 2010. Plant regeneration in spanish cedar, Cedrela odorata L., using zygotic embryo explants from mature seed and improvement of embryogenic nodule initiation by heat shock. In vitro Cellular and Development Biology - Plant 46(2): 126-133.

Da Costa-Nunes, E.; Volkmer de Castilho, C.; NettoMoreno, F.; Viana, A.M. 2002. In vitro culture of Cedrela fissilis Vellozo (Meliaceae). Plant Cell, Tissue and Organ Culture 70:259-268.

Daquinta, M.; Cid, M.; Lezcano, Y.; Pina, D.; Rodríguez, R. 2004. Formación de callos a partir de inflorescencias inmaduras en cedro y caoba híbrida. Biotecnología Vegetal 4(2): 121-124.

De la Torre, A.; López, C.; Yglesias, E.; Cornelius, J.P. 2008. Genetic (AFLP) diversity of nine Cedrela odorata populations in Madre de Dios, southern Peruvian Amazon. Forest Ecology and Management 255: 334-339.

García-Gonzáles, R.; Delgado, M.; Gonzáles, Y.; Gonzáles, A.; Garriga, M.; Caligari, P.; Carrasco, B.; Quiroz, K. 2011. In vitro propagation of Cedar (Cedrela odorata L.) from juvenile shoots. Chilean Journal of Agricultural Research 71(3): 376-382.

Gonzáles-Rodríguez, J.A.; Peña-Ramírez, Y.J. 2007. Establishment of efficient protocols for massive propagation of tropical trees from Mesoamerica through somatic embryogenesis: Cedrela odorata, Swietenia macrophylla, Cybistax donell-smithii, Crescentia cujete and Cordia dodecandra. Acta Horticulturae 748: 229-235.

Jiménez-Terry, F.; Barbón, R.; La O, M. Pérez, M.; Collado, R.; Acosta-Suárez, M.; Alvarado-Capó, Y.; Agramonte, D. 2007. Efecto de la revigorización en el establecimiento in vitro de ápices y segmentos nodales de Cedrela odorata L. Biotecnología Vegetal 7 (1): 45-51.

Muñoz, S. 2003. Embriogénesis somática en cedro (Cedrela odorata Linnaeus) a partir de cotiledones. Tesis de pre-grado, Universidad Nacional Agraria La Molina, Facultad de Ciencias, La Molina, departamento de Lima, Perú. $111 \mathrm{pp}$.

Murrieta, C.J.F. 2010. Influencia del morfotipo, fitohormona y sustrato en la propagación de estacas juveniles de Cedrela odorata L. (cedro colorado) en Pucallpa, Perú. Tesis de pre-grado, Universidad Nacional de Ucayali, Facultad de Ciencias Forestales y Ambientales, Pucallpa, departamento de Ucayali, Perú. 102 pp.

Patiño, F. 1997. Recursos genéticos de Swietenia y Cedrela en los neotrópicos: Propuestas para acciones coordinadas. Organización de las Naciones Unidas para la Agricultura y la Alimentación. Roma, Italia. 58 pp.

Peña-Ramírez, Y.J.; Juárez-Gómez, J.; GómezLópez, L.; Jerónimo-Pérez, J.L.; GarcíaSheseña, I.; González-Rodríguez, J.A.; Robert, M.L. 2010. Multiple adventitious shoot formation in Spanish Red Cedar (Cedrela odorata L.) cultured in vitro using juvenile and mature tissues: an improved micropropagation protocol for a highly valuable tropical tree species. In vitro Cellular and Development Biology-Plant 46(2): 149-160.

Pérez, J. Mesén, F. Hilje, L. Aguilar, M.E. 2002. Desarrollo de un método de micropropagación aplicable a genotipos selectos de Cedrela odorata L.: Optimización de la fase de multiplicación. Revista Forestal Centroamericana 38: 67-71.

Pérez, J.; Mesén, F.; Hilje, L.; Aguilar, M.E. 2006. Desarrollo de un método de micropropagación aplicable a genotipos selectos de Cedrela odorata: Fases de desarrollo y enraizamiento. Recursos Naturales y Ambiente 46-47:146-151.

Vila, A.; Gonzáles, A.; Rey, H.; Mroginski, L. 2009. Somatic embryogenesis and plant regeneration in Cedrela fissilis. Biologia Plantarum 53 (2): 383-386. 